\title{
Searches for dark matter and dark energy produced in association with a jet with the ATLAS detector
}

\author{
Jack Lindon* \\ on behalf of the ATLAS Collaboration \\ University of Birmingham (Great Britain) \\ E-mail: jack.henry.1indon@cern.ch
}

\begin{abstract}
The astrophysical and cosmological evidence of dark matter and dark energy provides some of the most compelling clues to the nature of physics beyond the Standard Model. If it interacts weakly with the Standard Model, dark matter can be produced directly at the LHC. Dark energy may exist in the form of light scalar particles that could contribute to the accelerating expansion of the observable universe. The following shows the results of the search for dark matter and the first collider search for dark energy, using events with large missing transverse momentum produced in association with jets using $36.1 \mathrm{fb}^{-1}$ of $\sqrt{s}=13 \mathrm{TeV} p p$ collision ATLAS data. This search sets the most stringent constraints on the suppression scale of disformal couplings of dark energy to Standard Model matter in the context of an effective field theory of dark energy following the Horndeski theory framework.
\end{abstract}

XXIX International Symposium on Lepton Photon Interactions at High Energies - LeptonPhoton2019 August 5-10, 2019, Toronto, Canada

\footnotetext{
*Speaker.
} 


\section{Introduction}

Despite the success of the Standard Model (SM), there are a number of measurements, particularly in the astrophysical and cosmological sector, that indicate the existence of Beyond the Standard Model (BSM) physics. Two of the largest indications of BSM physics in these sectors are dark matter and dark energy.

The rotational speed of stars in galaxies and gravitational lensing imply BSM physics [1-3]. These observations require either changes to the standard $r^{-2}$ scaling of gravitational force or more unseen non-luminous matter than regular matter, dubbed dark matter. Recent observations of galaxy cluster collisions [4], cannot be explained by any non-standard gravitational force that scales with observed mass, demonstrating the observation of dark matter.

The observation of the accelerated expansion of the universe (dubbed dark energy) requires BSM physics. The first observation of dark energy came from measurements of supernovae $[5,6]$. Recent observations come in the form of precision measurements of the cosmic microwave background [7] and the large scale structure of the universe [8].

Dark matter interacts with SM matter with low cross-sections, prohibiting direct detection with ATLAS. Instead, dark matter production is constrained by its influence on SM particles produced with it at the LHC. One approach used by the monojet search measures all final state particles from a collision and calculates the vector sum of the transverse momentum $\left(p_{\mathrm{T}}\right)$. As the initial protons that collide have $p_{\mathrm{T}} \approx 0$, a large total $p_{\mathrm{T}}$ in the final state indicates the production of undetected particles carrying missing transverse momentum $\left(E_{\mathrm{T}}^{\text {miss }}\right)$, allowing constraints to be placed on dark matter models. Selected monojet events have $E_{\mathrm{T}}^{\text {miss }}>250 \mathrm{GeV}$ along with 1-4 jets with at least one jet having $p_{\mathrm{T}}>250 \mathrm{GeV}$ and no identified leptons ( $e$ or $\mu$ ), a distinctive signature for BSM.

A Feynman diagram of a typical monojet event in association with dark matter is shown in Figure 1. In this diagram the jet arises from initial state radiation, allowing generic probing of BSM physics as it does not require that the jet directly interacts with BSM particles, only that they interact with at least one SM particle. In comparison to other $E_{\mathrm{T}}^{\text {miss }}+X$ searches, the monojet search has a large number of events from initial state radiation since $\alpha_{S} \gg \alpha_{E W}$.

\section{Models investigated}

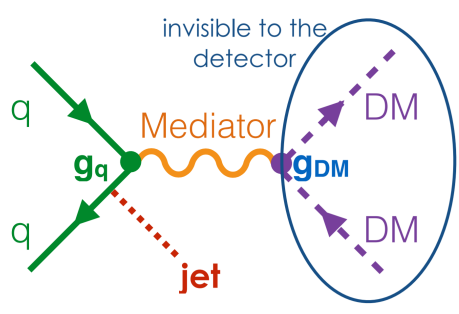

Figure 1: Monojet signature.

Weakly Interactive Massive Particles (WIMPs): The existence of a WIMP is often hypothesized [9], as it can lead to a relic density for non-relativistic matter in the early universe consistent with data from the Planck [10] and WMAP [11] Collaborations, if the mass is between a few $\mathrm{GeV}$ and one $\mathrm{TeV}$ and if it has electroweak-scale interaction cross sections. Limits for WIMPs pair produced in the $s$-channel via a mediator with axial-vector couplings, as in Figure 2, are shown in Figure 5a.

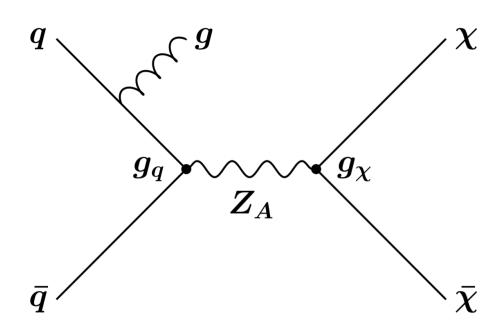

Figure 2: WIMP production. 
Pair production of Supersymmetric (SUSY) Squarks: Naturalness suggests 3rd-generation squarks have $m \lesssim 1 \mathrm{TeV}$ [12]. In R-parity conserving models [13], sparticles are pair produced and the lightest sparticle, assumed to be the lightest neutralino $\tilde{\chi}_{1}^{0}$, is stable. The results shown in Figure $5 \mathrm{~b}$ are interpreted in terms of stop squark pair production, where the stop squark decays into a charm quark and the lightest neutralino with a small $\Delta m \equiv m_{\tilde{t}}-m_{\tilde{\chi}_{1}^{0}}$. For $\Delta m \lesssim 25 \mathrm{GeV}$ both the $p_{\mathrm{T}}$ of the quark jets and the $E_{\mathrm{T}}^{\text {miss }}$ in the final state are small, making it difficult to fully reconstruct. However,

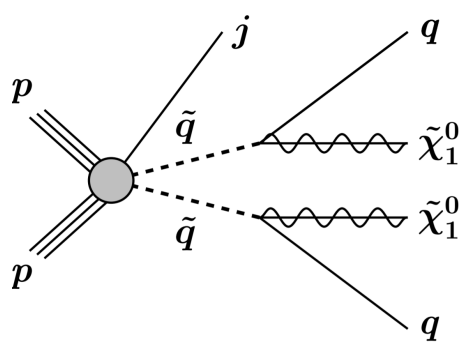

Figure 3: $\tilde{q} \tilde{q}$ production. initial state radiation can boost these final state particles, as in Figure 3, leading to larger $E_{\mathrm{T}}^{\mathrm{miss}}$, hence the monojet signature is ideal for probing small $\Delta m$.

Large Extra spatial Dimensions (LEDs): LEDs can explain the large difference between the EW scale and the Planck scale. In the ADD model of LEDs [14], the presence of $n$ LEDs of size $R$ leads to a fundamental Planck scale in $4+n$ dimensions given by $M_{P l}^{2} \sim M_{D}^{2+n} R^{n}$, where $M_{D}$ is the fundamental scale of the $4+n$-dimensional theory. $M_{D}$ is possibly on the order of $1 \mathrm{TeV}$, accessible at the LHC. In ADD SM particles are constrained to the usual spacetime dimensions, whereas gravitons can propagate through the LEDs, diluting gravity's strength. The LEDs are compactified resulting in massive graviton modes. Gravitons escaping into LEDs result in $E_{\mathrm{T}}^{\text {miss }}$ and a monojet signature; results are shown in Figure 5c.

Horndeski model of dark energy: Many dark energy models result in 5th forces that cause deviations in the $r^{-2}$ scaling of gravitational forces which laboratory experiments have probed [15]. For the 5th force to explain dark energy and satisfy existing constraints, it must be screened in regions of high density [16]. For dark energy fields mediated by a scalar, screening requires non-zero coupling between dark energy and SM fields, enabling LHC probing. A Horndeski theory [17], which provides the most general description of dark energy with a scalar field with second order equations of motion, has been developed [18]. Dark energy particle production is enhanced at high momentum transfers, making monojets an ideal search; results are shown in Figure 5d.

\section{Results}

The measured $E_{\mathrm{T}}^{\text {miss }}$ distribution in the monojet signal region is shown in Figure 4, in agreement with the SM background prediction. The error band shown in the ratio plot includes both the statistical and systematic uncertainties in the SM background predictions. For illustration overlaid are the $E_{\mathrm{T}}^{\mathrm{miss}}$ distribution of squarks, WIMPs, ADD and dark energy with free parameters set at values lying on the $95 \%$ exclusion contours of Figure 5.

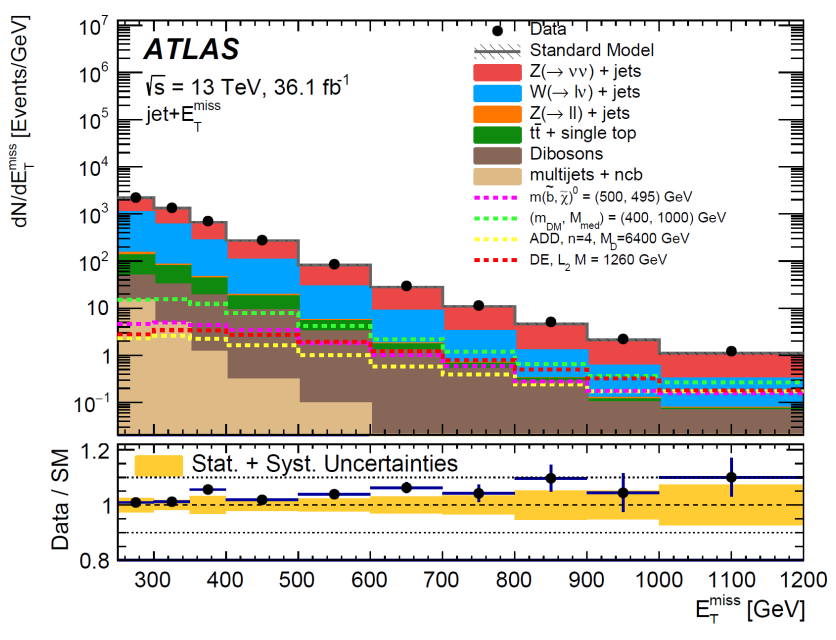

Figure 4: Measured $E_{\mathrm{T}}^{\text {miss }}$ compared to SM [19]. 


\section{Summary and future}

Searches for dark matter in the form of WIMPs and SUSY squarks, dark energy in the form of a new scalar field with coupling to gravitational and Standard Model fields, and large extra spatial dimensions in the monojet signature at the LHC using $36.1 \mathrm{fb}^{-1}$ of data collected by the ATLAS detector are reported [19, 20]. This search includes the first collider search for dark energy, which improves constraints on disformal dark energy from noncollider experiments by orders of magnitude.

HL-LHC statistics will greatly enhance discovery potential, extending the expected limit of Figure 5a to $m_{Z_{A}} \approx 2.6 \mathrm{TeV}$ with $3000 \mathrm{fb}^{-1}$ of

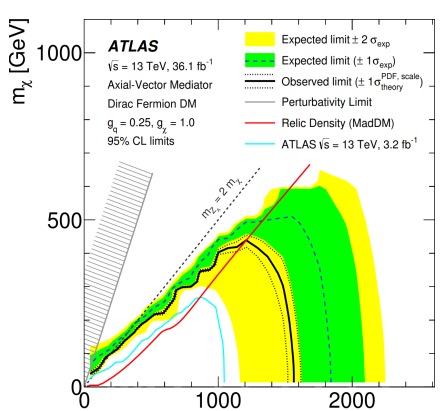

(a) WIMP. $\quad \mathrm{m}_{\mathrm{z}_{\mathrm{A}}}[\mathrm{GeV}]$

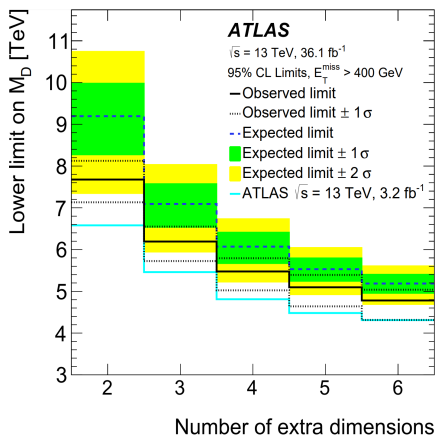

(c) ADD.

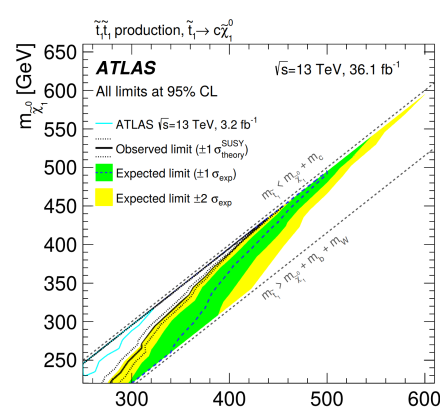

(b) SUSY.

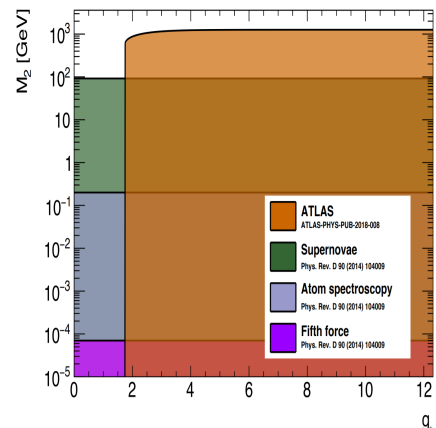

(d) Disformal dark energy.

Figure 5: 95\% CL exclusion contours[19, 20].

[21]. This can be further extended to $m_{Z_{A}} \approx 2.9 \mathrm{TeV}$ by a $50 \%$ reduction in theoretical systematics, while improvements to experimental systematics give negligible improvements to this model. A further 25-40\% increase in cross-section for this WIMP model is gained from $\sqrt{s}=13 \rightarrow 14 \mathrm{TeV}$.

\section{References}

[1] V. Trimble, Ann. Rev. Astron. Astrophys. 25 (1987) 425.

[2] G. Bertone, D. Hooper and J. Silk, Phys. Rept. 405 (2005) 279, arXiv: hep-ph/0404175 [hep-ph].

[3] J. L. Feng, Ann. Rev. Astron. Astrophys. 48 (2010) 495, arXiv: 1003.0904 [astro-ph.CO].

[4] D. Clowe et al., The Astrophysical Journal 648 (2006) 2, arXiv: astro-ph/0608407 [astro-ph].

[5] A. G. Riess et al., Astron. J. 116 (1998) 1009, arXiv: astro-ph/9805201 [astro-ph].

[6] S. Perlmutter et al., Astrophys. J. 517 (1999) 565, arXiv: astro-ph/9812133 [astro-ph].

[7] Planck Collaboration, Astronomy \& Astrophysics 594, A13 (2016) A13, arXiv: 1502.01589 [astro-ph.CO].

[8] S. Alam et al., The Astrophysical Journal Supplement Series 219, 12 (2015) 12, arXiv: 1501.00963 [astro-ph.IM].

[9] G. Steigman and M. S. Turner, Nucl. Phys. B 253 (1985) 375.

[10] R. Adam, et al., Planck Collaboration, Astron. Astrophys. 594 (2016) A1, arXiv: 1502.01582 [astro-ph.CO].

[11] G. Hinshaw et al., ApJS 208 (2013) 19, arXiv: 1212.5226 [astro-ph.CO].

[12] R. Barbieri and G. Giudice, Nucl. Phys. B 306 (1988) 63.

[13] P. Fayet, Phys. Lett. B 64 (1976) 159.

[14] N. Arkani-Hamed, S. Dimopoulos and G. Dvali, Phys. Lett. B 429 (1998) 263, arXiv: hep-ph/9803315 [hep-ph].

[15] ND. J. Kapner et al., Phys. Rev. Lett. 98 (2007) 021101, arXiv: hep-ph/0611184 [hep-ph].

[16] A. Joyce, B. Jain, J. Khoury and M. Trodden, Phys. Rept. 568 (2015) 1, arXiv: 1407.0059 [astro-ph.HE].

[17] G. W. Horndeski, International Journal of Theoretical Physics 10 (1974) 363.

[18] P. Brax, C. Burrage, C. Englert and M. Spannowsky Phys. Rev. D94 (2016) 084054, arXiv: 1604.04299 [hep-ph].

[19] ATLAS Collaboration, J. High Energ. Phys. (2018) 2018: 126, arXiv: 1711.03301 [hep-ex].

[20] ATLAS Collaboration, ATL-PHYS-PUB-2018-008.

[21] ATLAS Collaboration, ATL-PHYS-PUB-2018-043. 